\title{
Mutation in CSA creates a new photoperiod-sensitive genic male sterile line applicable for hybrid rice seed production
}

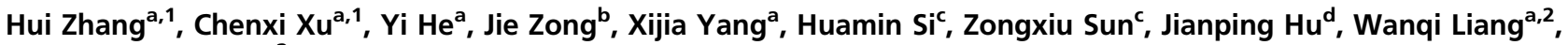 \\ and Dabing Zhang ${ }^{\mathrm{a}, 2}$
}

\begin{abstract}
${ }^{a}$ State Key Laboratory of Hybrid Rice, School of Life Sciences and Biotechnology, Shanghai Jiao Tong University, Shanghai 200240, China; ${ }^{b}$ Novel Bioinformatics Company, Shanghai 200240, China; 'China National Rice Research Institute, Hangzhou 310006, China; and ${ }^{\mathrm{d} D e p a r t m e n t}$ of Energy Plant Research Laboratory, Michigan State University, East Lansing, MI 48824
\end{abstract}

Edited by Qifa Zhang, Huazhong Agricultural University, Wuhan, China, and approved November 19, 2012 (received for review July 28, 2012)

\begin{abstract}
Rice is a major staple food worldwide. Making hybrid rice has proved to be an effective strategy to significantly increase grain yield. Current hybrid rice technologies rely on male sterile lines and have been used predominantly in indica cultivars. However, intrinsic problems exist in the implementation of these technologies, such as limited germplasms and unpredictable conversions from sterility to fertility in the field. Here, we describe a photoperiod-controlled male sterile line, carbon starved anther (csa), which contains a mutation in an R2R3 MYB transcription regulator of pollen development. This mutation was introduced into indica and japonica rice, and it rendered male sterility under short-day conditions and male fertility under long-day conditions in both lines. Furthermore, $F_{1}$ plants of csa and a restorer line JP69 exhibited heterosis (hybrid vigor), suggesting the feasibility of using this mutation to create hybrid rice. The csabased photoperiod-sensitive male sterile line allows the establishment of a stable two-line hybrid system, which promises to have a significant impact on agriculture.
\end{abstract}

PGMS line | hybrid breeding | sugar partitioning

$\mathbf{R}$ ice (Oryza sativa L.) is a major staple food that feeds more than one-half of the human population (1). The three- and two-line hybrid rice breeding technologies take advantage of heterosis (hybrid vigor) and have been successfully applied in many countries, leading to a more than $20 \%$ yield increase over inbred varieties (2). The three-line hybrid rice breeding system was developed in the 1970s after the revolutionary discovery of the wild abortive cytoplasmic male sterile cytoplasm (CMS-WA) for the breeding of CMS lines, which solved the problem of selfpollination in the varieties used for hybrid rice production (3). In this technology, a restorer line that contains the restorer gene(s) is used as the pollen donor to recover male fertility in $\mathrm{F}_{1}$ plants (hybrid rice) after its cross with the CMS line (Fig. S1A). To maintain CMS, a maintainer line, which has viable pollen grains and normal seed sets, is used as the pollinator to cross with the CMS line (Fig. S1 $A$ ). Although this system has been widely used for hybrid rice seed production, the requirement of a maintainer line with normal cytoplasm and a restorer line with nuclearencoded fertility restorer genes restricts breeders from exploiting more rice germplasms and complicates the procedures for generating hybrid seeds (4).

As a replacement of the three-line technology, a two-line system has been developed and applied for hybrid rice seed production after the discovery of the environmentally sensitive genic male sterile lines controlled by nuclear recessive gene(s). A japonica cultivar, Nongken 58S (NK58S), was the first spontaneous photoperiod-sensitive genic male sterile (PGMS) mutant identified (5). Because indica varieties are predominant in most rice planting regions, the PGMS loci/locus in NK58S were/was introduced into indica to generate breeding cultivars, such as Peiai 64S (PA64S) and Xin'an S. These indica lines frequently exhibit thermosensitive genic male sterility (TGMS) with a threshold of $\sim 24{ }^{\circ} \mathrm{C}(6)$. PGMS/TGMS lines can be used as the male sterile line or the maintainer line depending on the light and temperature conditions in the environment, and most rice cultivars can be used as the restorer line to restore the fertility in $\mathrm{F}_{1}$ hybrid plants (Fig. S1B). UDP-glucose pyrophosphorylase 1 cosuppressed rice exhibits a TGMS phenotype (7). Recently, a locus conferring PGMS/TGMS in NK58S and its derived lines was identified as a noncoding RNA gene, but the underlying molecular mechanisms for its function remain unclear $(8,9)$.

The two-line hybrid technology has obvious advantages, including a simplified breeding and seed production procedure. In addition, a wider range of restorers can be used (i.e., basically, all rice cultivars are capable of restoring the fertility in $\mathrm{F}_{1}$ hybrids) (10). However, the two-line hybrid technology for indica hybrid seed production frequently suffers from unpredictable conversions from sterility to fertility in the male sterile lines because of temperature changes in the field, which is problematic to hybrid seed production (10).

Given the limitation of currently used male sterile lines, exploring new male sterile lines is critical to improving hybrid rice technologies. Recent advances in rice functional genomic research have provided great opportunities to reveal additional beneficial alleles for breeding (11). Previously, we reported the identification of Carbon Starved Anther (CSA), an R2R3 MYB transcription factor that regulates sugar partitioning from photosynthetic tissues (sources) to anthers (sinks) to promote pollen maturation (12). The csa mutant, which contains a substitution of guanine with cytosine and one deletion of guanine in the first exon of $C S A$ and displays male sterility under short-day (SD) conditions, was identified from a japonica cultivar, 9522 (12). Here, we report the finding that this csa mutation renders male fertility under long-day (LD) conditions in both japonica and indica rice backgrounds, and it can be used to create new and more stable PGMS lines for two-line hybrid rice seed production.

\section{Result}

csa Is a Photoperiod-Sensitive Genic Male Sterile Mutant. Consistent with our previous report (12), when the csa mutant initiated panicle development during late August to early September in a

Author contributions: W.L. and D.Z. designed research; H.Z., C.X., Y.H., X.Y., H.S., Z.S., and W.L. performed research; H.Z., C.X., Y.H., J.Z., W.L., and D.Z. analyzed data; and J.H., W.L., and D.Z. wrote the paper.

The authors declare no conflict of interest.

This article is a PNAS Direct Submission.

Freely available online through the PNAS open access option.

${ }^{1}$ H.Z. and C.X. contributed equally to this work.

${ }^{2}$ To whom correspondence may be addressed. E-mail: zhangdb@sjtu.edu.cn or wqliang@ sjtu.edu.cn.

This article contains supporting information online at www.pnas.org/lookup/suppl/doi:10 1073/pnas.1213041110/-/DCSupplemental. 
paddy field in Shanghai, with a photoperiod of 12.5-13.0 h (SD), it had white and smaller anthers at the heading stage and displayed complete male sterility (Fig. S2 $A, B, G$, and $H$ ). However, when the initiation of panicle development occurred in late July to early August, during which time the photoperiod is $13.5-14.0 \mathrm{~h}$ (LD), the morphologies of anthers and pollen grains in csa were mostly normal (Fig. S2 $C, D, I$, and $J$ ). To verify this phenotype in a different location, we grew the mutants in a paddy field in Sanya, a region in southern China that is warmer and suitable for rice growing in the winter. In Sanya, when the rice panicle development initiated in early March, during which time the photoperiod is $12.0-12.5 \mathrm{~h}$ (SD), the csa mutant also displayed male sterility (Fig. S2 $E, F, K$, and $L$ ). These phenotypes were observed consistently from 2006 to 2010 in both locations, suggesting that the male sterility in csa may be controlled by photoperiod.

In our previous study, when csa was back-crossed with the wild-type (WT) cultivar 9522, the $\mathrm{F}_{2}$ population displayed an approximate $3: 1$ segregation ratio of fertile to sterile $(419: 126)$ under the SD conditions in Sanya when panicle development started in the winter, and all of the male sterile lines examined were homozygous for the csa mutation (12). In this study, we observed that the segregated mutants homozygous for the csa mutation were all fertile in Shanghai under LD conditions, indicating that the csa mutation cosegregates with a photoperiod-controlled male fertility trait. Moreover, the complemented transgenic lines of $c s a$, which contained a 4.3-kb WT CSA genomic DNA fragment, displayed normal male fertility under both LD and SD conditions (12), confirming that $C S A$ dysfunction results in the PGMS trait in the japonica cultivar, 9522.

To determine whether photoperiod regulates the fertility of $\operatorname{cs} a$, we manually changed the photoperiods by adjusting the length of light treatment in the paddy field in Shanghai. Under natural SD conditions, in contrast to the WT (Fig. $1 A, D, G$, and $J)$, csa had white and smaller anthers and completely aborted pollen grains, was male-sterile (Fig. $1 B, E$, and $H$ ), and eventually, had no seed set in the panicles (Fig. $1 K$ ). To convert SD to LD, lighting time in the paddy field was extended with artificial light from August 18 to August 27 in 2010, after the initial stage of panicle development in the mutant lines (Table S1). Under these conditions, csa exhibited close-to-normal anther and pollen grain morphologies (e.g., $80.1 \pm 5.57 \%$ normal pollen grains in mutant vs. $95.7 \pm 4.79 \%$ in the WT) and seed setting comparable with the seed setting of the WT (Fig. $1 C, F, I$, and $L$ ).

It has been reported that the sensitive time of fertility transition in PGMS/TGMS lines, such as NK58S, occurs during panicle development, when the secondary branch differentiates and
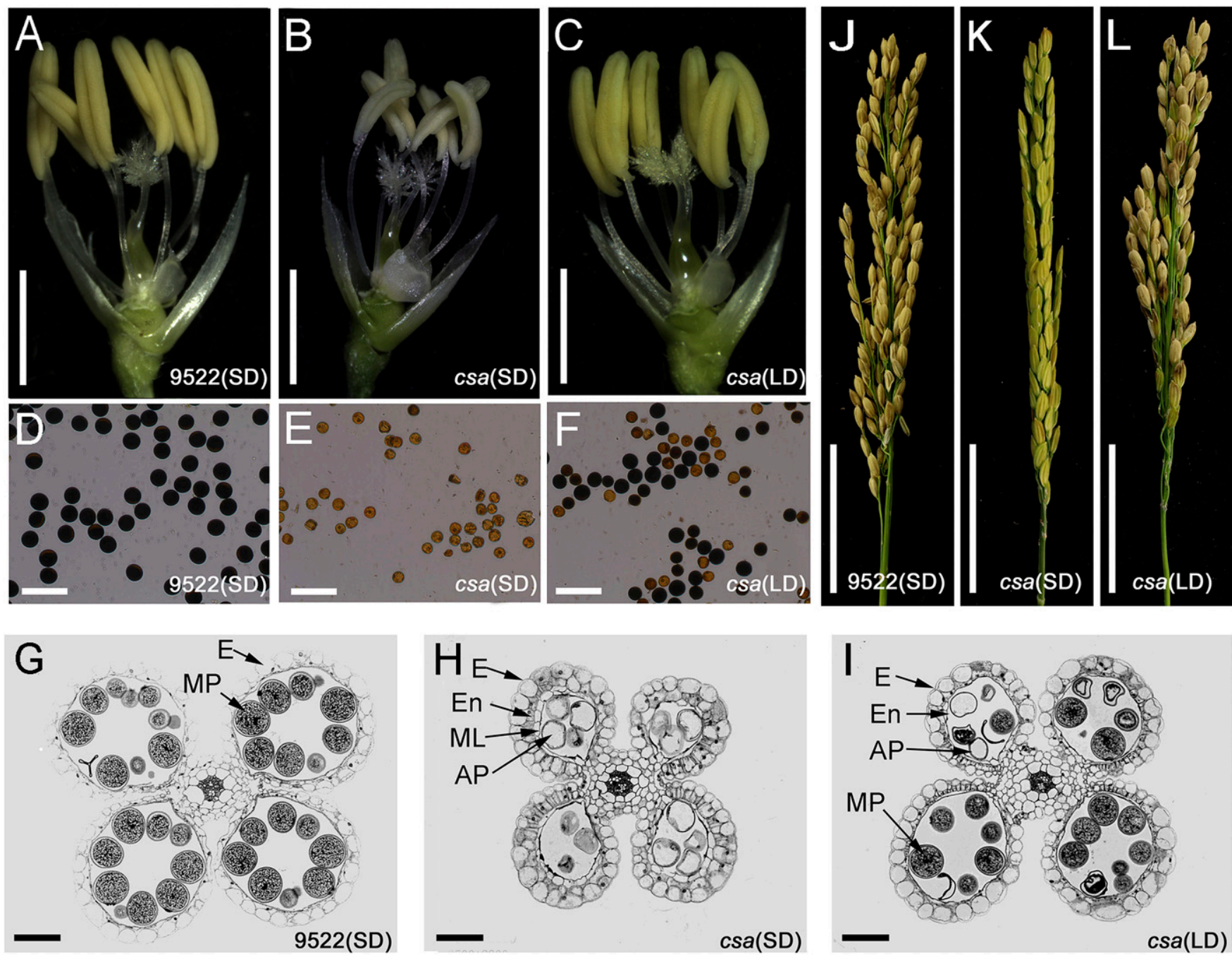

Fig. 1. csa phenotypes. $(A-C)$ Spikelet with the palea and lemma removed. $(D-F)$ Pollen grains stained with $I_{2}-K I$. (G-I) Transverse sections of anther locules. $(J-L)$ Panicles showing seed setting. AP, aborted pollen; $E$, epidermis; En, endothecium; ML, middle layer; MP, mature pollen. (Scale bars: $A-C, 2 \mathrm{~mm} ; D-F, 100$ $\mu \mathrm{m} ; \mathrm{G}-I, 50 \mu \mathrm{m}$; and $J-L, 5 \mathrm{~cm}$.) 
microsporogenesis takes place $(6,13)$. To check the critical stage for male fertility conversion in $\operatorname{csa}$, correlation analysis between seed setting and the time of LD treatment (Tables S1, S2, and S3) was performed using the SPSS statistical analysis software. Our results showed that the recovery of male fertility in csa correlated well with the LD treatment that took place $22-17 \mathrm{~d}$ before the heading stage; during this period, the secondary branch primordia are formed, and stamen and pistil are initiated (Fig. S3 and Tables S1, S2, and S3).

To further confirm the PGMS nature of $c s a$, we grew the mutants in growth chambers under various photoperiods and a daily average temperature of $24{ }^{\circ} \mathrm{C}$ or $28^{\circ} \mathrm{C}$ (Fig. S4 and Table $\mathrm{S} 4)$, conditions frequently used for PGMS mutant characterizations (14). Irrespective of the ambient temperature, the csa mutant was completely male-sterile under the 11.5 - or 12.5 -h photoperiod conditions (Fig. S4 $A-D$ and Table S4) and exhibited recovery of pollen fertility under 13.5 - and 14.5 -h photoperiod conditions (Fig. S4 $E-H$ and Table S4).

These results together suggest that the fertility of csa can be rescued by longer photoperiod and that csa is a PGMS line, which seems to be capable of overcoming the problems of instable male sterility caused by unpredictable temperature changes in current TGMS lines.

Defect in Sugar Partitioning in csa Was Recovered Under LD Conditions. Starch, as an energy reserve for pollen germination, accumulates during rice pollen maturation and thus, serves as a marker for pollen maturity (15). We showed previously that, under SD conditions, csa has defective assimilate allocation (i.e., sugar and starch contents are increased in leaves and reduced in anthers during pollen maturation) (Fig. $2 A$ and $B$ and Table S5) (12). Under LD conditions, however, this defect was partially rescued in csa. At stage 13 of anther development, when mature pollen grains form, starch levels increased $\sim 120 \%$ in the anther and decreased $42 \%$ in leaves compared with csa under SD conditions (Fig. $2 \mathrm{~A}$ and $B$ and Table S5). To measure the amount of sugars in the anther sink tissue, we used a ${ }^{14} \mathrm{C}$-sucrose feeding assay on excised stems that contained leaves and panicles on which the anthers were at developmental stages 11 and 13. The csa mutant had increased accumulation of ${ }^{14} \mathrm{C}$-labeled sugars in anthers under LD conditions compared with the same tissue under SD conditions (Fig. $2 C$ ). In agreement with this result, the expression of OsMST8, a monosaccharide transporter encoding gene that is critical for rice pollen starch accumulation and regulated by CSA (12), displayed a twofold increase in csa anthers under LD conditions compared with the SD conditions (Fig. $3 A$ ). These data collectively suggest that the deficiencies in sugar partitioning in csa can be rescued by LD conditions.
Differential Expression of CSA in SD and LD Conditions. To begin to understand the mechanism underlying the photoperiod-controlled conversion of male fertility in csa, we analyzed the expression patterns of $C S A$ in the WT under SD and LD conditions. Quantitative RT-PCR (qRT-PCR) was performed with RNA collected from anthers at stage 9, when microspores are released from the tetrad. Under SD conditions, $C S A$ is highly expressed in anthers, where its transcript level is $\sim 6.7$-fold higher than the level under LD conditions (Fig. $3 B$ ), suggesting that $C S A$ may play a key role in male fertility under SD conditions and have a less important function in pollen development under LD conditions.

We next performed microarray analysis of RNA from stage 9 anthers of csa to determine the level of rescue by LD at the global gene expression level and explore whether there are genes that may function preferentially in LD to replace the function of $C S A$. In SD, 880 genes were up-regulated and 455 genes were down-regulated in csa compared with the WT (Fig. $3 C$ and Dataset S1). Consistent with the recovery of male fertility in LD in $c s a$, the expression of 908 of 1,335 genes was completely or partially restored to WT levels under LD conditions in the mutant (Fig. $3 C$ and Dataset S1). Notably, two CSA homologs, OsMYB5 (Os05g41166) and OsMYB8 (Os01g45090), showed twoto threefold expression in LD vs. SD conditions in WT anthers (Fig. $3 D$ and $E$ ). In addition, in csa anthers, the expression level of 392 genes (including OsMYB5) is higher in LD than SD conditions (Fig. $3 C$ and Dataset $\mathrm{S} 1$ ). It remains to be elucidated whether these two CSA homologs or other proteins can function as the replacement of $C S A$ in male reproductive development under LD conditions.

Applicability of csa in Conferring PGMS in indica and Breeding. Strains used for hybrid rice production are mainly indica cultivars. The original NK58S line is a japonica line displaying PGMS. However, indica cultivars such as PA64S, into which the loci from NK58S were introgressed, predominantly exhibit TGMS rather than PGMS $(16,17)$. To determine whether introducing the csa mutation into indica cultivars can reproduce the phenotype that it confers in japonica, we crossed csa with the indica rice Zhenshan 97, a variety widely used in rice breeding. In the progeny, we selected male sterile lines homozygous for the csa mutation and displaying typical Zhenshan 97 morphological traits, such as longer leaf and purple spikelet awn and stigma (Fig. $4 A-C$ and Fig. S5 $A-G$ ). After two backcrosses with indica and two self-pollinations, $\mathrm{BC}_{2} \mathrm{~F}_{3}$ lines homozygous for csa also exhibited male sterility in SD conditions and recovered male fertility in LD conditions (Fig. $4 A-G$ ). These phenotypes are identical to those phenotypes of $c s a$ in the japonica background, suggesting that csa can produce new PGMS lines suitable for two-line hybrid rice breeding in both india and japonica. These results confirmed that $C S A$ is a PGMS gene.
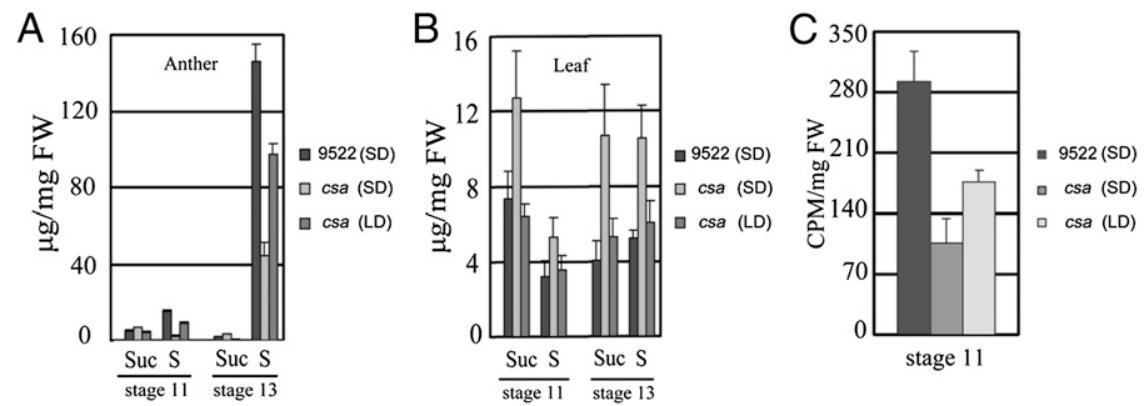

Fig. 2. Sugar and starch levels and ${ }^{14} \mathrm{C}$-signal accumulation in the flag leaf and anthers in the WT and csa. ( $A$ and $B$ ) Sucrose and starch levels at stages 11 and 13 in anthers $(A)$ and the flag leaf $(B) .(C){ }^{14} C$-signal accumulation in stage 11 anthers of the WT, csa under SD, and CSa under LD. The data are given as means \pm SE $(n=3)$. cpm, unit for radioactivity; FW, fresh weight; S, starch; Suc, sucrose. 
A

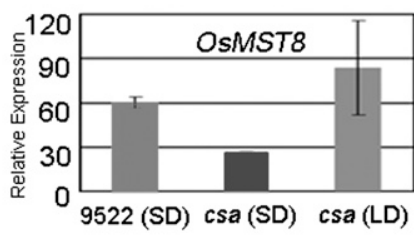

$\mathrm{B}$
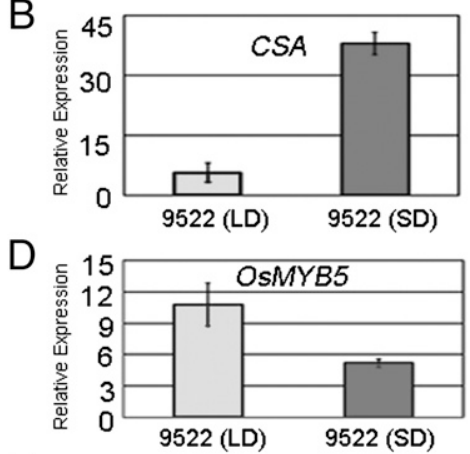

$\mathrm{E}$

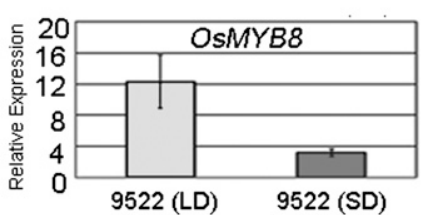

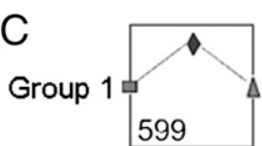
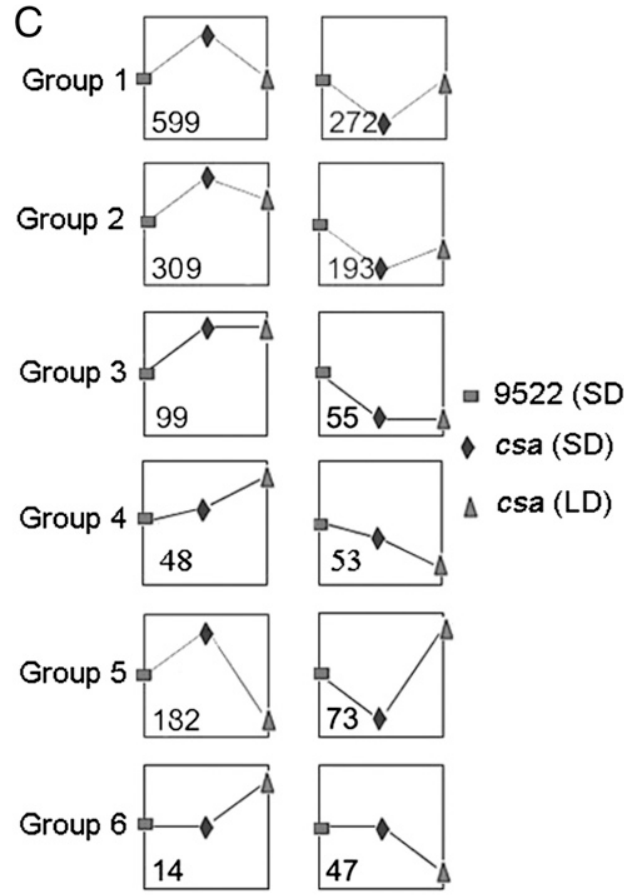

Fig. 3. Expression of OsMST8, CSA, OsMYB5, and OsMYB8 and analysis of genes with expression changes in csa. (A) Expression levels of OsMST8 in stage 9 anthers in WT (9522) and csa under SD and LD conditions. ( $B, D$, and E) Expression levels of CSA, OsMYB5, and OsMYB8 in WT (9522) stage 9 anthers under SD and LD conditions. (C) Clustering analysis of genes showing significant differential expressions in the anthers of WT (9522) under SD conditions and csa under SD and LD conditions. The candidate genes were categorized into six groups according to their different expression levels in stage 9 anthers of WT (SD), csa (SD), and csa (LD). Numbers on the graph indicate the number of hybridizing probes for each gene on the chip. Detailed information of the genes is listed in Dataset S1. GO analysis showed that these genes are mainly related to photosynthesis, light harvesting (GO: 0009765), lipid metabolic process (GO:0006629), response to abiotic stimulus (GO:0009628), and reproduction (GO:0000003) (Dataset S2). In $A, B, D$, and $E$, error bars indicate SD. Each reaction has three qPCR biological replicates.

The feasibility of csa in breeding was tested using csa as a female parent to cross with JP69, a japonica restorer line. $\mathrm{F}_{1}$ plants displayed normal male fertility (Fig. $4 H$ and $I$ and Fig. S5 $H-J$ ) and a strong hybrid vigor over JP69, such as significant increases in tiller number $(77.2 \%, n=20)$, grain number $(\sim 52.9 \%, n=10)$, and 1,000-grain weight $(12.9 \%, n=5)$ (Fig. $4 J-L)$. Our data confirmed the applicability of $c s a$ in hybrid rice production.

\section{Discussion}

PGMS/TGMS lines are valuable germplasm resources for crop hybrid breeding. Since the discovery of the first spontaneous photoperiod-sensitive genic male sterile mutant NK58S, a series of TGMS has been developed and applied for two-line hybrid rice production in countries such as China, India, Philippines, and the United States (18). One of these TGMS lines, PA64S, predominantly displays a temperature-controlled conversion from male sterility to fertility. It is male-sterile when anther development occurs at temperatures higher than $23.5^{\circ} \mathrm{C}$ and male-fertile when the temperature is in the range of $\sim 21-23{ }^{\circ} \mathrm{C}(16,17)$. Because of unpredictable environmental temperature changes, failure to produce hybrid rice seeds frequently occurs in TGMS in the field (10). In contrast to the fluctuating environmental temperatures, photoperiod is relatively stable in the field within a season; thus, PGMS is more preferred in hybrid rice breeding.

In this study, we showed that csa and its derived lines can be used as PGMS lines in the two-line hybrid rice breeding system. The csa mutation confers stable photoperiod-controlled male fertility in both indica and japonica, which is distinct from the currently used PGMS/TGMS lines. Until now, two types of rice PGMS lines have been reported. One type, such as NK58S (japonica), displays male sterility under LD and male fertility under SD (5). The other type, such as YiD1S and D52S, shows male sterility under SD and male fertility under LD, and it is called reverse PGMS (19, 20). The genetic loci governing the photoperiod-sensitive male fertility of YiD1S and D52S remain to be determined at the molecular level $(19,20)$. In our work, introduction of the csa mutation into indica rice Zhenshan 97 rendered male sterility under SD conditions and male fertility under LD conditions, suggesting that csa belongs to the reverse PGMS category and can produce new PGMS lines suitable for twoline hybrid rice breeding in both indica and japonica. Thus, the csa mutation has a great potential in two-line hybrid rice breeding, overcoming some of the problems in the current hybrid rice systems, such as limited germplasm use and unstable male sterility, and it may greatly benefit large-scale crop breeding systems.

Plant male reproductive development is controlled by the interaction between genetic components and environmental signals such as photoperiod and temperature. This study suggests that photoperiod regulates the expression of $C S A$, which encodes a key regulator of sugar partitioning during anther development. Previously, we showed that, in late anther development, CSA plays a key role in promoting sugar partitioning from the source tissue leaf to the sink tissue anther by directly regulating the expression of OsMST8, which encodes a monosaccharide transporter (12). Monosaccharide transporters are capable of transporting various monosaccharides across membrane barriers, and rice OsMST8 is a key component of the specialized anther apoplastic sugar transport pathway (21-23). The mutation in CSA caused a dramatic reduction of OsMST8 expression in anthers and reduced amount of sugar accumulation in the anther under SD conditions, leading to male sterility (Figs. 2 and 3A) (12). In contrast, OSMST8 expression and the sugar level in csa 

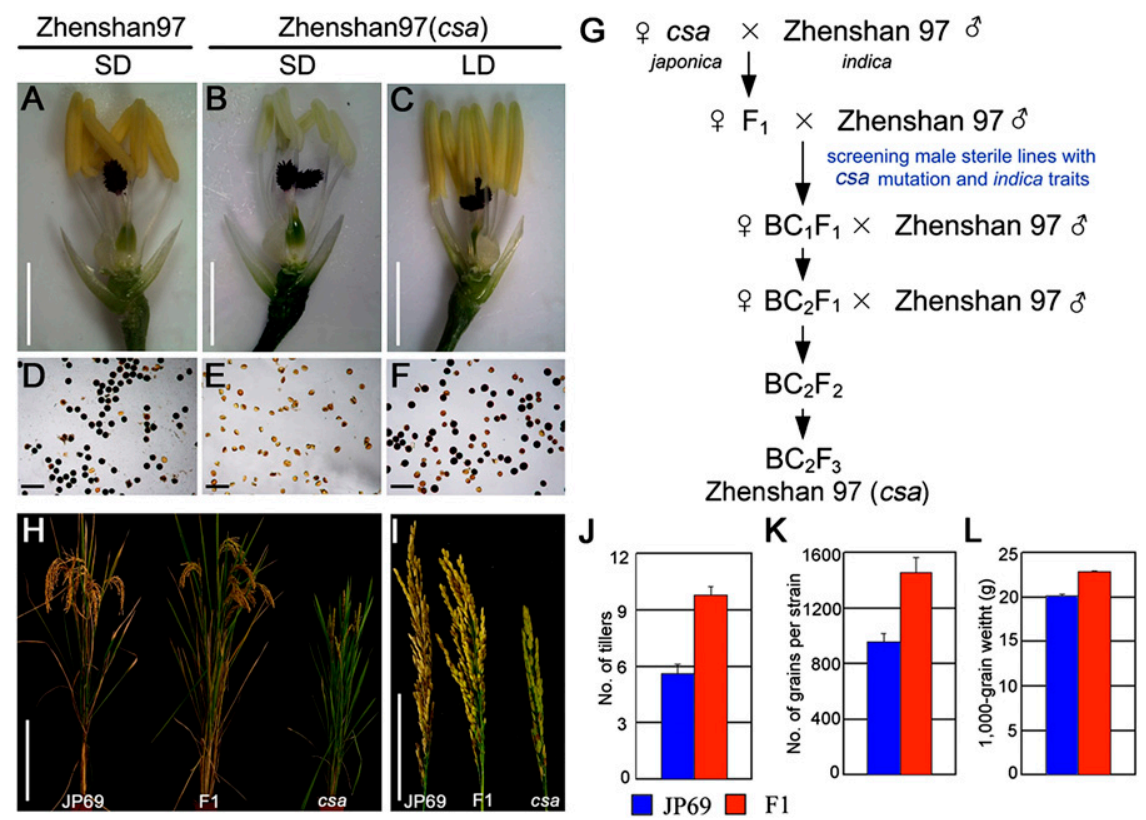

Fig. 4. Phenotypes of Zhenshan 97 with or without csa, JP69, and hybrid $F_{1}$ lines. $(A-C)$ Spikelet after the palea and lemma are removed. ( $\left.D-F\right)$ Pollen grains stained with $\mathrm{I}_{2}-\mathrm{KI}$. (G) Strategy for screening csa in the indica background. $(H)$ Morphologies of whole plant. (I) Panicles. $(J-L)$ Comparison of the number of tillers $(J)$ and grains $(K)$ per plant with 1,000-grain weight $(I)$.

anthers was recovered nearly to the normal level under LD conditions (Figs. 2 and $3 A$ ). We hypothesize that CSA plays a less important role in pollen development under LD conditions. In support of this hypothesis, $C S A$ expression in $\mathrm{SD}$ conditions is about 6.7-fold higher than in LD conditions (Fig. $3 B$ ).

Our genome-wide analysis indicated that, in csa under LD conditions, the expression of a large number of genes with expression that is changed under SD conditions was completely or partially restored to WT levels. CSA is completely dysfunctional in the csa mutant (12); therefore, other genes may be mainly responsible for regulating anther development under LD conditions. Candidates for these regulators include the two CSA homologs, OsMYB5 and OsMYB8, which exhibit higher expression levels in LD conditions (Fig. $3 D$ and $E$ and Dataset S1). OsMYB5 is closely related to another Arabidopsis R2R3 MYB gene, $M Y B 33$, and double mutant between myb33 and another myb mutant (myb65) displays conditional male sterility with reduced male fertility under low light intensity or higher temperatures (24). OsMYB8 is closely related to Arabidopsis MYB21, which encodes a flower-specific transcription factor that affects anther development (25). Other genes with higher expression in LD than SD could also act in a CSA-independent regulatory pathway of anther development in the LD.

In summary, this work identified csa as a causal mutant displaying a PGMS trait. The csa mutant and derived csa-containing lines overcome some of the problems in the current hybrid rice systems and will potentially be greatly beneficial to large-scale crop breeding programs.

\section{Methods}

Materials and Growth Conditions. The varieties 9522 (O. sativa L. ssp. japonica), Zhenshan 97 (O. sativa L. ssp. indica), csa, Zhenshan 97 (csa), and JP69 (O. sativa L. ssp. japonica) were used for this study. csa was generated by treating rice seeds 9522 with ${ }^{60} \mathrm{Co} \gamma$-ray radiation (26). Zhenshan 97 (csa) has the csa mutation in the Zhenshan 97 background, which was generated by backcrossing csa with the indica variety Zhenshan 97 . The hybrid rice was bred by crossing JP69 (male) and csa (female) in Sanya (18_N, 109_E) and later grown in Shanghai (30_N, 121_E).

Experiments in the paddy fields were carried out at two sites, Sanya and Shanghai, China, from 2006 to 2010 in normal rice growing seasons. To confirm the PGMS nature of csa, photoperiods were extended by illuminating rice plants (after panicle initiation) with artificial light with a $200-\mathrm{W}$ incandescent source (lamp) that is $\sim 50 \mathrm{~cm}$ above the rice panicles. We measured the natural photoperiod length according to the website record: www.time.ac.cn/serve/sun_time/sh.htm.

Experiments in the growth chambers were carried out in China National Rice Research Institute, with the mean temperature of $24^{\circ} \mathrm{C}$ or $28^{\circ} \mathrm{C}$, photoperiod of $11.5,12.5,13.5$, or $14.5 \mathrm{~h}$, and $70 \%$ relative humidity. When each csa seedling had five leaves on the stem, SD (10 h light/14 h dark) treatment was conducted to promote the transition from vegetative growth to reproductive growth. After $10 \mathrm{~d}$ of SD treatments for flowering induction, the rice plants were moved into growth chambers for photoperiod treatments (Table S4). Rice anther staging was described in the work by Zhang et al. (27).

Phenotype Characterization and Fertility Examination. Plants were photographed with a Nikon E995 digital camera and a Leica S8 APO stereo microscope (Leica Microsystems). For transverse section analysis of anther locules, anthers were collected and fixed as described (28). Semithin $(4 \mu \mathrm{m})$ sections were made using a Leica RM 2265 programmable rotary microtome (Leica Microsystems) and stained with $0.05 \%$ Toluidine Blue (28). Transverse sections were photographed using a Leica DM2500 microscope (Leica Microsystems). To analyze pollen fertility, anthers of five mature flowers from each plant at heading time were collected. Pollen grains were then stained with $1 \%$ potassium iodide solution $\left(\mathrm{I}_{2}-\mathrm{KI}\right)$ and photographed using the Leica DM2500 microscope.

Sugar and Starch Measurement and ${ }^{14} \mathrm{C}$-Signal Accumulation. WT and csa (SD and LD) anthers and flag leaves were obtained at stages 11 and 13 of anther development for sucrose and starch measurement and analysis using previously published methods (12). To measure ${ }^{14} \mathrm{C}$-signal accumulation, WT and csa panicles from about 100 flowers at stage 11 of anther development were excised and treated with radiolabeled [fructose-U- ${ }^{14} \mathrm{C}$ ]sucrose. Radioactivity (cpm) was measured as previously described (12).

qPCR Assay. Total RNA was isolated from rice anthers at stage 9 with the TRIzol Reagent Kit (Invitrogen) treated with DNase and then reverse-transcribed as previously described (12). qRT-PCR was performed with a Bio-Rad CFX96 RealTime PCR Detection System using SYBR Green I. Samples were normalized using ACTIN1 expression; the relative expression levels were measured using the $2^{(-\Delta C t)}$ analysis method. Primer sequences used are listed in Table S6.

Microarray Analysis. Total RNA was isolated from stage 9 anthers in csa under LD and SD conditions and stage 9 WT anthers under SD using TRIzol (Invitrogen). The microarray experiment was performed with Affymetrix rice 
genome array by Gene Tech Biotechnology Company using the standard Affymetrix protocol (Affymetrix). Significance analysis of microarray was used to identify differentially expressed genes. Genes with at least a threefold change in expression, $P$ values $<0.001$, and false discovery rate $<0.05$ were chosen for additional analysis.

The microarray data were also verified by qRT-PCR using independently generated stage 9 samples. Eight genes, which have more than threefold changes in expression (Fig. S6), were selected for qRT-PCR. The fold changes observed from qRT-PCR closely mirrored those changes obtained from the microarray analysis, confirming the reliability of the microarray data.

Gene Ontology Analysis. The Gene Ontology (GO) annotations of microarray genes were downloaded from National Center for Biotechnology Information (www.ncbi.nlm.nih.gov), UniProt (www.uniprot.org), and GO (www. geneontology.org). The elim Fisher algorithm was used to do the GO enrichment

1. Delseny M, et al. (2001) Rice genomics: Present and future. Plant Physiol Biochem 39(3-4):323-334.

2. Cheng SH, Zhuang JY, Fan YY, Du JH, Cao LY (2007) Progress in research and development on hybrid rice: A super-domesticate in China. Ann Bot (Lond) 100(5): 959-966.

3. Yuan L (1986) Hybrid rice in China. Chin J Rice Sci 1(1):8-18.

4. Chen L, Xiao Y, Lei D (2010) Mechanism of sterility and breeding strategies for photoperiod/thermo-sensitive genic male sterile rice. Rice Sci 17(3):161-167.

5. Shi M (1985) The discovery and preliminary studies of the photoperiod-sensitive re cessive male sterile rice (Oryza sativa L. subsp. japonica). Sci Agric Sin 18(2):44-48.

6. Mou T, et al. (2001) Characterization of fertility alteration in 8 environment-sensitive genic male-sterile lines of indica Rice. Acta Bot Sin 43(2):238-242.

7. Chen $R$, et al. (2007) Rice UDP-glucose pyrophosphorylase 1 is essential for pollen callose deposition and its cosuppression results in a new type of thermosensitive genic male sterility. Plant Cell 19(3):847-861.

8. Ding J, et al. (2012) A long noncoding RNA regulates photoperiod-sensitive male sterility, an essential component of hybrid rice. Proc Natl Acad Sci USA 109(7): 2654-2659.

9. Zhou H, et al. (2012) Photoperiod- and thermo-sensitive genic male sterility in rice are caused by a point mutation in a novel noncoding RNA that produces a small RNA. Cell Res 22(4):649-660.

10. Zhang Q, et al. (1994) Using bulked extremes and recessive class to map genes for photoperiod-sensitive genic male sterility in rice. Proc Natl Acad Sci USA 91(18): 8675-8679.

11. Jiang $Y$, et al. (2012) Rice functional genomics research: Progress and implications for crop genetic improvement. Biotechnol Adv 30(5):1059-1070.

12. Zhang H, et al. (2010) Carbon Starved Anther encodes a MYB domain protein that regulates sugar partitioning required for rice pollen development. Plant Cell 22(3): 672-689.

13. Yuan S, Zhang Z, Xu C (1988) Studies on the critical stage of fertility change induced by light and its phase development in HPGMR. Acta Agron Sin 14(1):7-12.

14. Yu Y, Si H, Hu G, Fu Y, Sun Z (2006) Analysis on fertility transformation reaction to photothermo treatments of Peiai64S from different sources. Sci Agric Sin 39(5):1064-1068.

15. Datta R, Chourey PS, Pring DR, Tang HV (2001) Gene-expression analysis of sucrosestarch metabolism during pollen maturation in cytoplasmic male-sterile and fertile lines of sorghum. Sex Plant Reprod 14(3):127-134. test, because it can iteratively remove the genes mapped to significant GO terms from more general (higher-level) GO terms; therefore, the significant GO terms will not be overshadowed by the general GO terms (29). Gene ontology categories with a $P$ value $<0.01$ were selected (Dataset S2).

ACKNOWLEDGMENTS. This article is dedicated to the late Professor Mengmin Hong, who passed away on November 13, 2012. He gave helpful advice to our work. The authors acknowledge Zhijing Luo, Mingjiao Chen, and Zhaolu Meng for genetic analysis. This work was supported by funds from National Natural Science Foundation of China Grant 30830014; National Key Basic Research Developments Program, Ministry of Science and Technology, China Grants 2013CB126902 and 2011CB100101; the 863 Hitech Project, Ministry of Science and Technology, China Grants 2011AA10A101 and 2012AA10A302; Science and Technology Commission of Shanghai Municipality Grants 10JC1406400, 10DZ2294100, and 11JC1404900; and National Transgenic Major Program Grants 2011ZX08009-003-003 and 2009ZX08009-108B (to D.Z. and W.L.).

16. Xu M, Zhou G, Chen L (1999) Response of fertility of Pei'ai 64 S to temperature and photoperiod in rice. Acta Agron Sin 25(6):772-776.

17. Lu C, Zou J, Hu N, Yao K (2007) Plant temperature for sterile alteration of a temperature-sensitive genic male sterile rice, Peiai64S. Sci Agric Sin 40(1):19-26.

18. Yuan L (2004) Hybrid rice technology for food security in the world. Crop Res 18(4): 185-186.

19. Peng HF, et al. (2008) Molecular mapping of two reverse photoperiod-sensitive genic male sterility genes (rpms1 and rpms2) in rice (Oryza sativa L.). Theor Appl Genet 118 (1):77-83.

20. Joseph CA, Chen Z, Ma D, Zeng $H$ (2011) Analysis of short photo-periodic sensitive genic male sterility and molecular mapping of rpms3 ( $t$ ) gene in rice (Oryza sativa L.) using SSR markers. Genes Genomics 33(5):513-519.

21. Oliver SN, Dennis ES, Dolferus R (2007) ABA regulates apoplastic sugar transport and is a potential signal for cold-induced pollen sterility in rice. Plant Cell Physiol 48(9): 1319-1330.

22. Mamun EA, Alfred S, Cantrill LC, Overall RL, Sutton BG (2006) Effects of chilling on male gametophyte development in rice. Cell Biol Int 30(7):583-591.

23. Oliver SN, et al. (2005) Cold induced repression of the rice anther specific cell wall invertase gene OSINV4 is correlated with sucrose accumulation and pollen sterility. Plant Cell Environ 28(12):1534-1551.

24. Millar AA, Gubler F (2005) The Arabidopsis GAMYB-like genes, MYB33 and MYB65, are microRNA-regulated genes that redundantly facilitate anther development. Plant Cell 17(3):705-721

25. Shin B, et al. (2002) AtMYB21, a gene encoding a flower-specific transcription factor, is regulated by COP1. Plant $J 30(1): 23-32$.

26. Chen $\mathrm{L}$, et al. (2006) Isolation and genetic analysis for rice mutants treated with $60 \mathrm{Co}$ $\gamma$-Ray. J Xiamen Univ 45(1):82-85.

27. Zhang $D$, Luo $X$, Zhu $L$ (2011) Cytological analysis and genetic control of rice anther development. J Genet Genomics 38(9):379-390.

28. Li N, et al. (2006) The rice Tapetum Degeneration Retardation gene is required for tapetum degradation and anther development. Plant Cell 18(11):2999-3014.

29. Alexa A, Rahnenführer J, Lengauer T (2006) Improved scoring of functional groups from gene expression data by decorrelating $\mathrm{GO}$ graph structure. Bioinformatics 22 (13):1600-1607. 